\title{
Beitrag zur Kenntnis der Absorption von Gasen durch Kohle;
}

\author{
ivon \\ Wilhelm Vaubel.
}

In einer Reihe von Abhandlungen, die in meinem Lehrbuche der theoretischen Chemie zusammengefabt sind, habe ich gezeigt, da $B$ bei gewissen Vorgängen ein Teil der chemischen Affinität in Wirkung tritt, den ich mit dem Namen Gravitoaffinität bezeichnete. Es gelten hierfür folgende Sätze:

1. Die Gravitoaffinität ist der Teil der chemischen Affinität, deren Wirksamkeit durch die Masse bezw. durch das Gewicht des betreffenden Atoms oder Moleküls bedingt ist.

2. Die Gravitoaffinität zeigt in ihrer Wirksamkeit dasselbe Verhalten wie die Gravitation, d. h. die Gravitoaffinität ist direkt proportional der Masse. Ob sie umgekehrt proportional der Entfernung ist, bedarf erst noch der Untersuchung.

3. Der Beweis für die direkte Proportionalität von Gravitoaffinität und Gewicht ist durch die Untersuchungen über die Dissoziationswärmen sowie die Reaktionswärmen bei Ausfällungen erbracht worden.

Beispiele waren:

$$
\begin{aligned}
& \mathrm{J}_{2}=2 \mathrm{~J} \\
& \mathrm{~N}_{2} \mathrm{O}_{4}=2 \mathrm{NO}_{2} \\
& \mathrm{Cu}_{2}=\mathrm{Cu}-\mathrm{Cu} \\
& \mathrm{Hg}_{2}=\mathrm{Hg}-\mathrm{Hg}
\end{aligned}
$$

\begin{tabular}{|c|c|c|}
\hline \multirow[b]{2}{*}{ Mol.-Gew. } & \multicolumn{2}{|c|}{ Dissoziationswärme } \\
\hline & beobachtet & berechnet \\
\hline 254 & 285 & 285 \\
\hline 92 & 106 & 104,9 \\
\hline $1 / 263 \times 2$ & 72 & 72 \\
\hline $1 / 2200 \times 2$ & 228 & 228 \\
\hline & \multicolumn{2}{|c|}{ Reaktionswärme } \\
\hline $\begin{array}{c}\text { Mol.Gew. } \\
235\end{array}$ & $\begin{array}{c}\text { beobachtet } \\
264\end{array}$ & $\begin{array}{c}\text { berechnet } \\
263.7\end{array}$ \\
\hline 188 & 210,9 & 197,7 \\
\hline 143,5 & 158 & 161 \\
\hline 232 & 296 & 260,8 \\
\hline 15,7 & 132 & 107,2 \\
\hline
\end{tabular}

$$
\begin{aligned}
& \mathrm{Ag}+\mathrm{J}=\mathrm{AgJ} \\
& \mathrm{Ag}+\mathrm{Br}=\mathrm{AgBr} \\
& \mathrm{Ag}+\mathrm{Cl}=\mathrm{AgCl} \\
& \mathrm{Hg}+\mathrm{S}=\mathrm{HgS} \\
& \mathrm{Cu}+\mathrm{S}=\mathrm{CuS}
\end{aligned}
$$


Vaubel: Absorption von Gasen durch Koble. 233

Die unter "berechnet" angeführten Werte sind durch Multiplikation des Molekulargewichtes mit dem Gravitoaffinitätsfaktor 1,12 erbalten worden.

Bei der Absorption von Gasen durch Koble wird Wärme frei. Es lag nahe, diese Absorptionswärme ebenfalls auf die Wirkung der Gravitoaffinität zu beziehen, da es sich bei diesem Vorgang doch allem Anschein nach um eine sog. molekulare Anlagerung handelt.

Wie man schon früher bestrebt war, durch Vergleich dieser Absorptionswärmen mit der Verflüssigungs- oder Lösungswärme eine Erklärung für das Auftreten der Absorptionswärme zu finden, zeigen z. B. die Arbeiten von Favr e. ${ }^{1}$ ) Diese Vergleiche führten zu keinem Resultate. Legen wir aber die oben angeführten Sätze über die Gravitoaffinität zugrunde, so können wir mit Hilfe des Gravitoaffinitätsfaktors bemerkenswerte Resultate erhalten.

Nachstehend seien zunächst die Beobachtungen von Chappuis ${ }^{2}$ ) über die Absorption von verschiedenen Gasen durch Holzkohle wiedergegeben, soweit sie hier in Frage komme. Angewandt wurden immer je $2.85 \mathrm{~g}$ Holzkohle, und die Beobachtungen der Absorptionswärmen wurden für die verschiedensten Volumina durchgeführt. Es wurden beobachtet für $1 \mathrm{ecm}$ ron

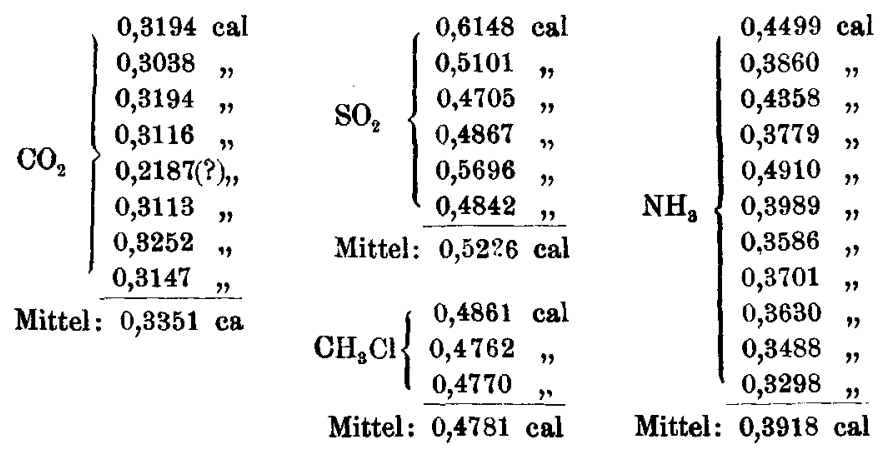

AuBerdem liegen noch folgende Beobachtungen von Favre (a. a. O.) vor. Die auf $1 \mathrm{~g}$ kondensiertes Gas entwickelten Wärmemengen betragen für

1) Favre, JB. 1874, S. 110. Ann. Chim. 1, 209 (1874).

2) Chappuis, JB. 1883, S. 129. 
234 Vaubel: Absorption von Gasen durch Kohle.

$\begin{array}{llll}\mathrm{HCl} & 274 \mathrm{cal} & \mathrm{HJ} & 173 \mathrm{cal} \\ \mathrm{HBr} & 191 " & \mathrm{~N}_{2} \mathrm{O} & 169 "\end{array}$

Gehen wir nun von der Voraussetzung aus, daß bei dieser Absorption allein die Gravitoaffinität in Wirksamkeit tritt, so können wir mit Hilfe des Gravitoaffinitätsfaktors und der gemachten Beobachtungen die Größe der zu erwartenden Molekularverbindung berechnen.

Es ergibt sich:

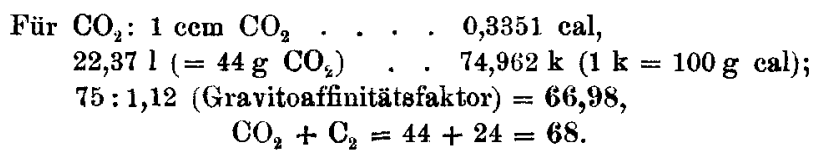

Danach tritt je 1 Grammolekül $\mathrm{CO}_{2}$ mit 2 Atomen Kohlenstoff in Bindung.

$$
\begin{aligned}
& \text { Für } \mathrm{SO}_{2}: 1 \mathrm{ccm} \mathrm{S}_{2} \text {. . . } 0,5226 \mathrm{cal} \text {, } \\
& 64 \mathrm{~g} \mathrm{SO}_{2} \text {. . . } 116,9 \mathrm{k} \text {, } \\
& 116,9: 1,12=104,4 \text {, } \\
& \mathrm{SO}_{2}+\mathrm{C}_{3}=64+36=100 \text {, } \\
& \mathrm{SO}_{2}+\mathrm{C}_{4}=64+48=112 .
\end{aligned}
$$

Für $\mathrm{CH}_{s} \mathrm{Cl}: 1 \mathrm{cem} \mathrm{.} \mathrm{.} \mathrm{.} \mathrm{0,4781} \mathrm{cal,}$

$$
\begin{aligned}
50,5 \mathrm{~g} \cdot & \cdot 106,95 \mathrm{k}, \\
106,95: 1,12 & =\mathbf{9 5 , 5} \\
\mathrm{CH}_{3} \mathrm{Cl}+\mathrm{C}_{3}=50,5+36= & \mathbf{8 6 , 5}
\end{aligned}
$$$$
\mathrm{CH}_{9} \mathrm{Cl}+\mathrm{C}_{4}=50,5+48=98,5
$$

Für $\mathrm{NH}_{\mathrm{a}}: 1 \mathrm{ccm}$. . . . $0,3918 \mathrm{cal}$,

$$
\begin{aligned}
& 17 \mathrm{~g} \cdot \cdot \cdot \quad 71,54 \mathrm{k}, \\
& 71,54: 1,12=63,8 \\
& \mathrm{NH}_{\mathrm{s}}+\mathrm{C}_{4}=17+48=65 .
\end{aligned}
$$

Für HCl: $1 \mathrm{~g}$. . . . . . $274 \mathrm{cal}$,

$$
36,5 \mathrm{~g} \cdot \text {. } 100,0 \mathrm{k} \text {, }
$$$$
100: 1,12=89,3
$$

$$
\mathrm{HCl}+\mathrm{C}_{4}=36,5+48=84,5 \text {. }
$$

Für HBr: $1 \mathrm{~g}$. . . . . $191 \mathrm{cal}$,

$$
81 \mathrm{~g} \cdot \text {. . . . } 154,7 \mathrm{k} \text {, }
$$

$$
154,7: 1,12=138 \text {, }
$$

$\mathrm{HBr}+\mathrm{C}_{4}=81+48=129$.

Für HJ : $1 \mathrm{~g}$. . . . . . $173 \mathrm{cal}$,

$$
\begin{aligned}
& 128 \mathrm{~g} \text {. . . } 221,4 \mathrm{k} \text {, } \\
& 221,4: 1,12=197,9 \text {, } \\
& \mathrm{HJ}+\mathrm{C}_{4}=128+48=176 .
\end{aligned}
$$


Vaubel: Absorption von Gasen durch Kohle. 235

$$
\begin{aligned}
& \text { Für } \mathrm{N}_{2} \mathrm{O}: 1 \mathrm{~g} \text {. . . . . . } 169 \mathrm{cal} \text {, } \\
& 44 \mathrm{~g} \text {. . . . . . } 74,36 \mathrm{k} \text {, } \\
& 74,36: 1,12=66,4 \text {, } \\
& \mathrm{N}_{2} \mathrm{O}+\mathrm{C}_{8}=44+24=68 \text {, } \\
& \mathrm{N}_{2} \mathrm{O}+\mathrm{C}_{3}=44+36=80 \text {. }
\end{aligned}
$$

Wenn man von kleinen Unstimmigkeiten absieht, die auch zum groBen Teil durch Fehler in den Beobachtungen bedingt sein mögen, läßt sich aus diesen Beobachtungen und Berechnungen schließen:

1. $\mathrm{DaB}$ bei der Absorption von $\mathrm{SO}_{2}, \mathrm{NH}_{3}, \mathrm{CH}_{3} \mathrm{Cl}, \mathrm{HCl}$ sich je 1 Grammolekül des Gases mit 4 Atomen $\mathrm{C}$ vereinigt, sowie daß bei der Absorption von $\mathrm{CO}_{2}$ und $\mathrm{N}_{2} \mathrm{O}$ sich je 2 Grammoleküle mit je 4 Atomen $\mathrm{C}$ vereinigen.

2. DaB die Kohlenstoffmolekel in dieser Kohlensorte aus 4 Atomen oder einem Vielfachen davon besteht.

Dieses Resultat ist in guter Übereinstimmung mit einer anderen von mir ausgeführten Berechnung, wonach die MolekulargröBe der amorphen Kohle sich zu 23,3 also $24 \mathrm{C}$ berechnet. ${ }^{1}$ )

3. DaB mithin auch die Absorption der Gase durch die Anzahl der Kohlenstoffatome in der Molekel begrenzt ist.

Da auf 24 Atome $=1$ Molekül Kohlenstoff 12 Moleküle $\mathrm{CO}_{2}$ und $\mathrm{N}_{2} \mathrm{O}$ und 6 Moleküle $\mathrm{NH}_{3}, \mathrm{SO}_{2}, \mathrm{CH}_{3} \mathrm{Cl}$ und $\mathrm{HCl}$ kommen, muß also eine entsprechende Zahl freier Valenzen in der Kohlenstoffmolekel vorhanden sein oder doch leicht eine entsprechende Umlagerung stattfinden können. Denkt man sich die Anlagerung von $\mathrm{CO}_{2}$ oder $\mathrm{N}_{2} \mathrm{O}$ in folgender Weise:

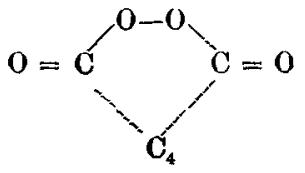

oder

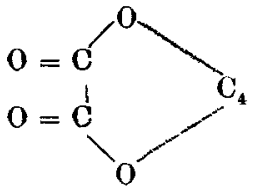

und die der anderen Gase nach beifolgenden Formeln<smiles>CC1CS(=O)(=O)C1</smiles><smiles>C#C[C]1CC(C)C1</smiles><smiles>[CH]1CC2CC1C2</smiles>

1) Vaubel, dies. Journ. [2] 55, 542 (1897). Zeitschr. angew. Chem. 13, 60, $520(1900)$ 'Theor. Chem. I, 381. 
236 Vaubel: Absorption von Gasen dureh Kohle.

so binden sich die letzteren direkt mit je 2 Valenzen an die Kohlenstoffmolekel, während $\mathrm{CO}_{2}$ und $\mathrm{N}_{2} \mathrm{O}$ sich erst aneinander lagern. Es würden also in der Kohlenstoffmolekel von 24 Atomen je 12 Valenzen für die Anlagerung der Gasmolekeln zur Verfügung stehen müssen.

Zum Schlusse sei noch darauf aufmerksam gemacht, daB auch die bisherigen Beobachtungen über die absoluten Mengen der absorbierten Gase mit diesen Ausführungen in guter Übereinstimmung stehen. So absorbieren z. B. 1,57 g Kohle im Maximum $178 \mathrm{ccm} \mathrm{NH}_{3}$. Nimmt man $80 \%$ reinen Kohlenstoff an, so ergeben sich hiernach für $48 \mathrm{~g}$ Kohlenstoff $6,2 \mathrm{~g} \mathrm{NH}_{3}$, während nach obiger Theorie $17 \mathrm{~g}$ aufgenommen werden können. Es ist aber niemals eine gröBere Aufnahme beobachtet worden, als der von mir entwickelten Annahme entspricht. 